\title{
Mechanical Properties of Polyamide 6 Reinforced Microfibrilar Composites
}

\author{
Nadya V. Dencheva, ${ }^{1}$ Maria J. Oliveira, ${ }^{1}$ Antonio S. Pouzada, ${ }^{1}$ Mark P. Kearns, ${ }^{2}$ Zlatan Z. Denchev ${ }^{1}$ \\ ${ }_{1}^{1}$ I3N-Institute for Polymers and Composites, University of Minho, campus Azurém, Guimarães 4800-058, Portugal \\ ${ }^{2}$ Queens University, School of Mechanical and Aerospace Engineering, Belfast BT9 5AG, UK
}

DOI 10.1002/pc.21056

Published online in Wiley Online Library (wileyonlinelibrary.com)

POLYM. COMPOS., 32:407-417, 2011

\section{ABSTRACT:}

The mechanical behavior of microfibrilar composites (MFC), consisting of a matrix of high-density polyethylene (HDPE) and reinforcement of polyamide 6 (PA6) fibrils, with and without compatibilization, was studied. The composites were produced by conventional processing techniques with various shape and arrangement of the PA6 reinforcing entities: long, unidirectional, or crossed bundles of fibrils (UDP and CPC, respectively), middlelength, randomly oriented bristles (MRB), or non-oriented micrometric PA6 spheres (NOM). The tensile, flexural, and impact properties of the MFC materials (UDP, CPC, and MRB) were determined as a function of the PA6 reinforcement shape, alignment and content, and compared with those of NOM, the non-fibrous composite.
It was concluded that the in-situ MFC materials based on HDPE/PA6 blends display improvements in the mechanical behavior when compared with the neat HDPE matrix, e.g., up to $33 \%$ for the Young modulus, up to $119 \%$ for the ultimate tensile strength, and up to $80 \%$ for the flexural stiffness.

POLYMER COMPOSITES 32:407-417, 2011.

(C) 2011 Society of Plastics Engineers

\section{INTRODUCTION}

Microfibrilar composites (MFC) are a special type of materials combining the easier processability of the glass fiber reinforced polymer composites with the presence of high aspect ratio fibril reinforcements with diameters in the nanometer range [1]. The preparation of MFC comprises three basic steps [2-4]. First, melt-blending is performed of two or more immiscible polymers with melting temperatures Tm differing by $308 \mathrm{C}$ or more. In the polymer blend so formed, the reinforcing phase should always originate from the higher melting component, and the matrix phase from the lower-melting component. Second, the polymer blend is drawn at temperatures above the glass transition $T_{g}$ of the two components leading to their orientation, also called fibrillation. Finally, selective isotropization by melting is induced in the matrix at a temperature below the Tm of the fibrils. This last step can be performed by compression molding of the oriented precursors [5-7]. Chopping the latter into pellets allows their reprocessing into MFC by extrusion or by injection molding. This alternative was reported by Monticciolo et al. [8] and was followed later by other authors [9-11] on different polymer blends.

\footnotetext{
The content of this article was orally presented in November 2008 as an integral part of the PhD thesis of NVD. Some of the tensile strength and flexural data were communicated by ZZD at the Polymer Processing Society Congress in Salerno, Italy, 2008 and at the PPSRegional Meeting in Cyprus in 2009.

Correspondence to: Z. Z. Denchev; e-mail: denchev@dep.uminho.pt

Contract grant sponsor: Fundação para a Ciência e Tecnologia (FCT); contract grant number: POCI/CTM/57358/2004.

Contract grant sponsor: Fundação para a Ciência e Tecnologia (FCT); contract grant number: SFRH/BPD/45252/2008 (to NVD).
}

Adhesion between the two components of the MFC is expected to play an important role in their mechanical behavior. There are two basic ways to manipulate the adhesion at the phase boundary in MFCs: (i) by creation of chemical bonds and (ii) through the formation of transcrystalline layers (TCL). In most MFC with potential for industrial application, the matrix phase does not possess the necessary chemical functionality, so as to be bonded chemically to the reinforcing phase; therefore, the introduction of a compatibilizer is required. In MFC based on high-density polyethylene (HDPE) matrices and polyamide 6 or 12 (PA6, PA12) microfibril reinforcements, good compatibilization was obtained when an HDPEmaleic anhydride (MAH) copolymer was used as a third component [12, 13]. Filippi et al. [14] described another compatibilizer for polyolefin/ polyamide blends based on ethylene-acrylic acid copolymers. In the case of polyestercontaining blends, alongside with $\mathrm{MAH}$, ethylene-glycidyl meth-acrylate copolymers have also been used [15].

Transcrystalline morphology has been registered in various types of MFC in the absence $[13,16,17]$ or in the presence [18] of chemical bonding between the matrix and reinforcing components.

Because of the high aspect ratio of the crystalline and oriented microfibrilar reinforcement, and in view of the various possibilities to strengthen the matrix-fibril interface by compatibilization or transcrystallization, the mechanical properties of the optimized MFC are superior to those of the corresponding neat matrix material [19]. 
TABLE 1. Composition of the HDPE/PA6/YP composites.

\begin{tabular}{lccc}
$\begin{array}{l}\text { HDPE/PA6/YP } \\
\text { composite designation }\end{array}$ & HDPE, wt $\%$ & PA6, wt $\%$ & YP, wt $\%$ \\
\hline $90 / 10 / 0$ & 90.0 & 10.0 & 0 \\
$80 / 20 / 0$ & 80.0 & 20.0 & 0 \\
$70 / 20 / 10$ & 70.0 & 20.0 & 10.0 \\
$75 / 20 / 5$ & 75.0 & 20.0 & 5.0 \\
$77.5 / 20 / 2.5$ & 77.5 & 20.0 & 2.5 \\
$65 / 30 / 5$ & 65.0 & 30.0 & 5.0 \\
\hline
\end{tabular}

From each composition UDP, CPC, MRB, and NOM composites were produced (Fig. 1).

YP, HDPE/MAH copolymer commercially available from DSM as Yparex.

UDP, MFC lamina obtained from continuous oriented cables arranged in the form of unidirectional ply.

CPC, MFC laminate obtained from cross-ply arranged oriented cables. MRB, MFC obtained from middle-length randomly distributed bristles.

NOM, composite obtained from nonoriented mixture.

Most of the mechanical studies on MFC were made with systems based on polyolefin matrices [low-density polyethylene (LDPE), HDPE, or polypropylene (PP)], reinforced by virgin of recycled poly(ethylene terephthalate) (PET) microfibrils. Thus, MFC obtained from LDPE/PET oriented blends selectively isotropized by injection molding achieved elastic moduli approaching those of $L D P E+30 \%$ glass fibers. The tensile strength of MFC has reached at least two times that of the neat LDPE matrix material, the impact strength of the MFC being $50 \%$ higher [16]. Extensive mechanical studies have also been performed with the PP/PET $[9,20]$ and HDPE/PET MFC [21, 22]. HDPE/PA12 MFCs have also been obtained and characterized in tensile mode [12], observing a $74 \%$ improvement of the ultimate strength and a maximum of $43 \%$ improvement in the Young's modulus, when compared with HDPE. It should be noted that these results were obtained with a single MFC (HDPE/PA12/YP $1 / 470 /$ $20 / 10$ wt \%), i.e., without any optimization of composition.

The tribological properties of polyolefin matrices reinforced by PET or PA6,6 were also studied [23]. It was established that the reinforcement with PA66 fibrils leads to higher wear resistance in comparison to PET in MFC with the same matrix material. The wear rates were found to be much lower in MFC with uniaxialy oriented reinforcing fibrils when compared to materials with random orientation of the reinforcements.

To the best of our knowledge, there is no detailed mechanical study on PA6 as reinforcing component in MFC. Apart from being cheaper than PA12, PA6 has significantly higher melting temperature, thus providing a larger processing window for the selective compression molding of the HDPE/PA6 blend, which is important for maintaining the oriented fibrilar morphology of the polyamide reinforcements. Some of the mechanical properties of PA6 (i.e., tensile and impact strengths) are reportedly higher than those of PA12 [24], thus expecting stronger reinforcing effect in the HDPE/PA6 MFC systems. In the present work, tensile, flexural, and impact tests were performed on various HDPE/PA6 MFC and the effects of the different compatibilization, HDPE/PA6 ratio, the form, and arrangement of the reinforcing entities on the mechanical behavior were studied.

\section{EXPERIMENTAL}

\section{Sample Preparation}

Oriented blends of PA6, HDPE, and HDPE-MAH copolymer [Yparex (YP)] with compositions shown in Table 1 were prepared by extrusion followed by cold drawing. An extensive description of the raw materials used and of the preparation of the MFC was already given elsewhere [13]. In summary, the processing conditions were chosen in a way that the PA6 fibril reinforcing phase could reach its best mechanical efficiency, as established in earlier studies $[25,26]$. The six oriented HDPE/PA6/ YP compositions in Table 1 were obtained initially in the form of continuous cables with diameter around $1 \mathrm{~mm}$. These cables were then cut to shape and compression molded at temperature below the melting point of PA6 into three MFC types: (i) in the form of orthotropic laminae obtained from unidirectional plies of cables (UDP), (ii) cross-ply laminates (CPC) obtained from two plies of oriented cables arranged perpendicularly, and (iii) composites from middle-size randomly distributed PA6 bristles (MRB). Compression molded non-oriented pellets obtained right after extrusion and denoted as "non-oriented material" (NOM) were also produced from each blend and tested for comparison. Figure 1 shows the visual aspect of various types of precursors. Figure 2 depicts the preparation of the CPC laminates from two perpendicularly aligned unidirectional plies of oriented cables.

\section{Microscopic Examination}

To analyze the morphology of the oriented precursors and of the final MFC, scanning electron microscopy (SEM) of freeze-fractured specimens was used. All samples were sputter-coated with gold and observed in a Leica Microsystems Cambridge Ltd., Cambridge, UK at magnifications of $2.0 \mathrm{k}, 5.0 \mathrm{k}$, and $7.5 \mathrm{k}$.

\section{Mechanical Tests}

The UDP MFC laminae were used for tensile tests. Impact strength and three-point flexural tests were performed on the CPC laminates. MRB and NOM composites were analyzed with the three mechanical tests. The data were compared with those of the neat HDPE matrix and/or the oriented PA6. The tensile tests were performed in an (Instron, High Wycomb, UK) at $23 \pm 2{ }^{\circ} \mathrm{C}$ with a standard load cell of $1 \mathrm{kN}$ at constant crosshead speed of 50 $\mathrm{mm} / \mathrm{min}$. Test samples with gauge length and width of 25 $\mathrm{mm}$ and $4 \mathrm{~mm}$, respectively, and thicknesses varying in the 1.1-1.5 $\mathrm{mm}$ range were cut out from each composite in two mutually perpendicular directions. Ten specimens of each sample were studied to calculate the average and the standard deviation. 


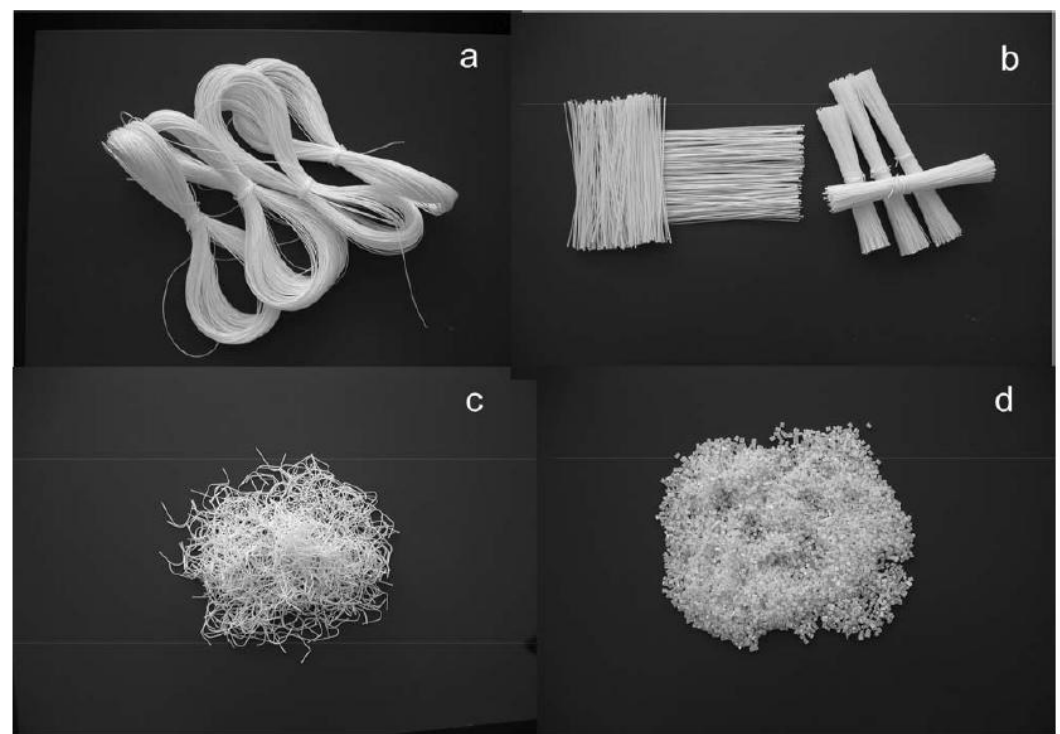

FIG. 1. Visual aspect of the MFC precursors: (a) infinite oriented cable; (b) bundles of parallel bristles; (c) MRB, middle-length randomly distributed bristles; and (d) NOM, granules obtained by palletizing the extrudate after the extruder die.

The nominal stress was determined as the ratio of the tensile force and the initial cross section of the sample. The nominal strain was determined as the ratio of the sample gauge length at any time during drawing and that before testing. The Young moduli were calculated from the stress/strain curves at $1 \%$ strain (secant modulus). For each UDP MFC sample, two experimental values for the Young modulus $E$ and the ultimate strength $\sigma_{\max }$ were obtained: longitudinal $\left(E_{1}, \sigma_{1 \max }\right)$ and transverse $\left(E_{2}, \sigma_{2 \max }\right)$. Theoretical predictions for these parameters were calculated as suggested in Ref. 27. The Eq. 1 was used for the $E_{1}$ values:

$$
E_{1}=V_{\mathrm{f}} E_{\mathrm{f}}+E_{\mathrm{p}}\left(1-V_{\mathrm{f}}\right) \approx V_{\mathrm{f}} E_{\mathrm{f}}
$$

where $E_{p}$ and $E_{f}$ are the respective moduli of the matrix and of the fibres, and $V_{f}$ is the volume fraction of fibres (the rule of mixtures). The transverse modulus $E_{2}$ estimates were derived from Eq. 2:

$$
\frac{1}{E_{2}}=\frac{V_{\mathrm{f}}}{E_{\mathrm{f}}}+\frac{1-V_{\mathrm{f}}}{E_{\mathrm{p}}}
$$

The longitudinal tensile strength of the UDP composites was estimated approximately by:

$$
\sigma_{1 \max }=\sigma_{\mathrm{f} \max } \cdot V_{\mathrm{f}}
$$

where $\sigma_{1 \max }$ is the strength of the PA6 oriented fibre.

In the transversal direction it is assumed that:

$$
\sigma_{2 \max }=0.33 \sigma_{\mathrm{p} \max }
$$

where $\sigma_{\mathrm{pmax}}$ is the tensile strength of the matrix.
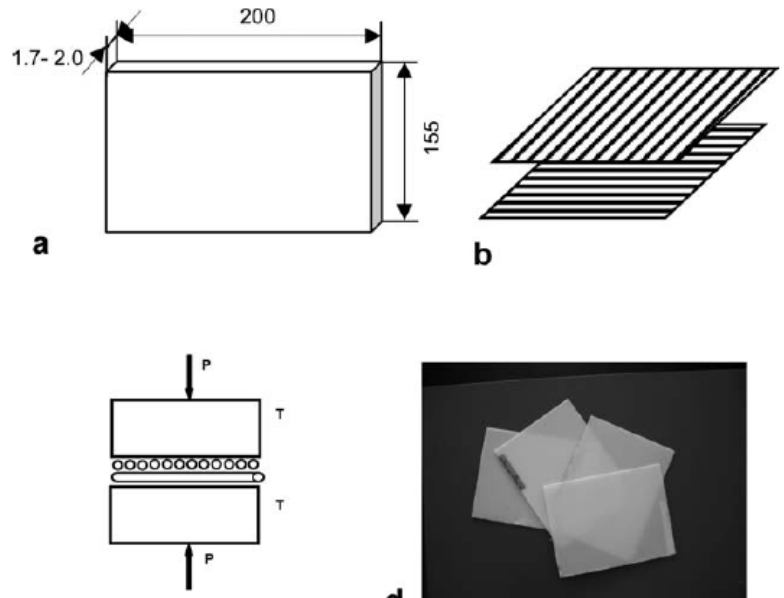

C

d

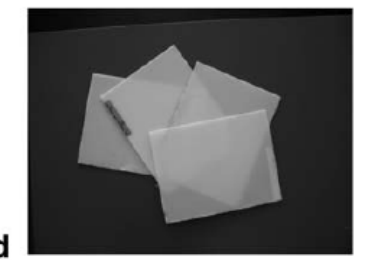

FIG. 2. Preparation of the cross-ply laminates (CPC): (a) dimensions of composite plates, mm; (b) two unidirectional plies of oriented precursors, perpendicularly aligned; (c) compression molding at temperature, $T=$ $160^{\circ} \mathrm{C}$, and pressure, $P=1.5 \mathrm{MPa}$; and (d) visual aspect of the resulting laminate plates used for flexural and impact resistance tests.

Flexural tests were performed by the three-point support test method used by Nunes et al. [28] as shown in Fig. 3. The support was mounted in the same Instron machine used for the tensile tests and this time operating in compression mode. Rectangular samples (155 mm x 100 $\mathrm{mm}$ ) were cut out from the CPC MFC plates and placed upon the support. A maximum load of $1 \mathrm{kN}$ was applied at the centre of the sample using a crosshead speed of 5 $\mathrm{mm} / \mathrm{min}$. From the force-displacement curves the slope $S_{p}$ was determined and used to calculate the reduced flexural stiffness $C_{R}[28]$ :

$$
C_{\mathrm{R}}=\frac{3}{2 \pi h^{3}} R^{2} S_{\mathrm{p}}
$$




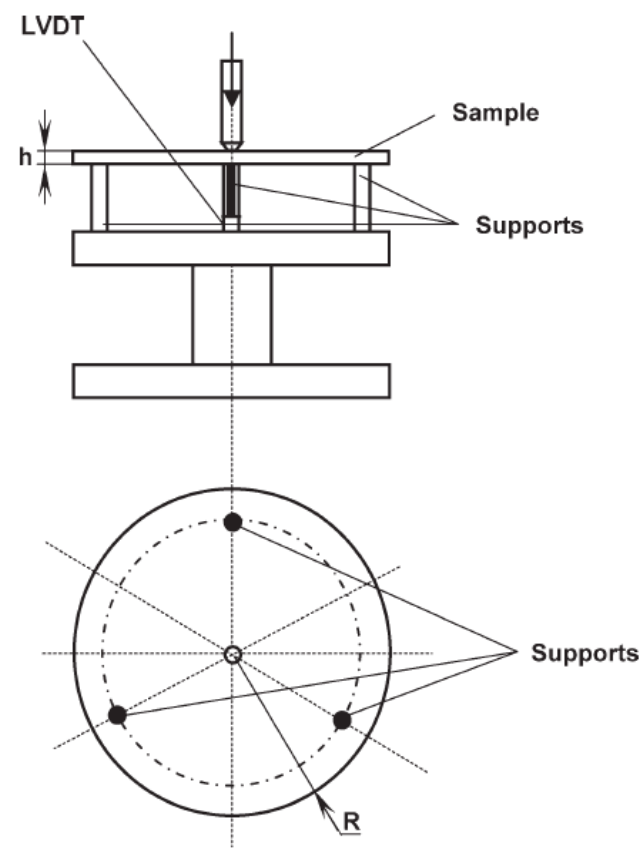

FIG. 3. Schematic diagram of the three-point support flexural test: $h$, sample thickness; LVDT, linear variable differential transformer displacement transducer; $R, 46.75 \mathrm{~mm}$.

In equation $3, \mathrm{~h}$ is the sample thickness varying in the 1.4 $1.8 \mathrm{~mm}$ range and $\mathrm{R}=46.75 \mathrm{~mm}$ is the radius of the circumference on which the three supports are located. Eight samples of each CPC, MRB, and NOM laminates were tested. Similar measurements were performed with the neat HDPE and the improvement factor, IF, was calculated as:

$$
\mathrm{IF}=\frac{C_{\mathrm{R}}^{\text {Comp }}-C_{\mathrm{R}}^{\text {Matrix }}}{C_{\mathrm{R}}^{\text {Matrix }}} \times 100[\%]
$$

In the other works where the three-point support test was used $[28,29]$, circular test specimens were tested. In this study, rectangular plates were used instead. To assess the deviations introduced by these geometries with respect to the theoretical test geometry, simulations with the ABAQUS software [30] were performed with two forces ( $1 \mathrm{kN}$ and $100 \mathrm{~N}$ ). The output of these simulations is resented in Fig. 4. Based on this, it is possible to conclude that using rectangular plates instead of overhanging circular plates has a negligible effect on the results.

Impact tests were performed using a CEAST USA Charlotte 28208 NC. The test samples were square plates of $60 \times 60 \mathrm{~mm}$ machined from the respective compression molded pates. Eight impact samples of each material were impact tested at $240^{\circ} \mathrm{C}$, the cooling being achieved by a mixture of liquid nitrogen and petroleum ether. The samples were impacted using a drop height of $1 \mathrm{~m}$, leading to an impact speed of approximately $4.4 \mathrm{~m} / \mathrm{s}$. The force experienced by the sample as a function of time is determined. Assuming a constant impact speed, time is recalculated as displacement. From the force versus displacement graphs, peak force, peak energy, and total energy were determined. In each test, the values for peak and total energy are divided by the sample thickness to give the specific energy per millimetre thickness. The latter values are considered as peak and total impact strengths, respectively, as suggested by Pick and Harkin- Jones [31].
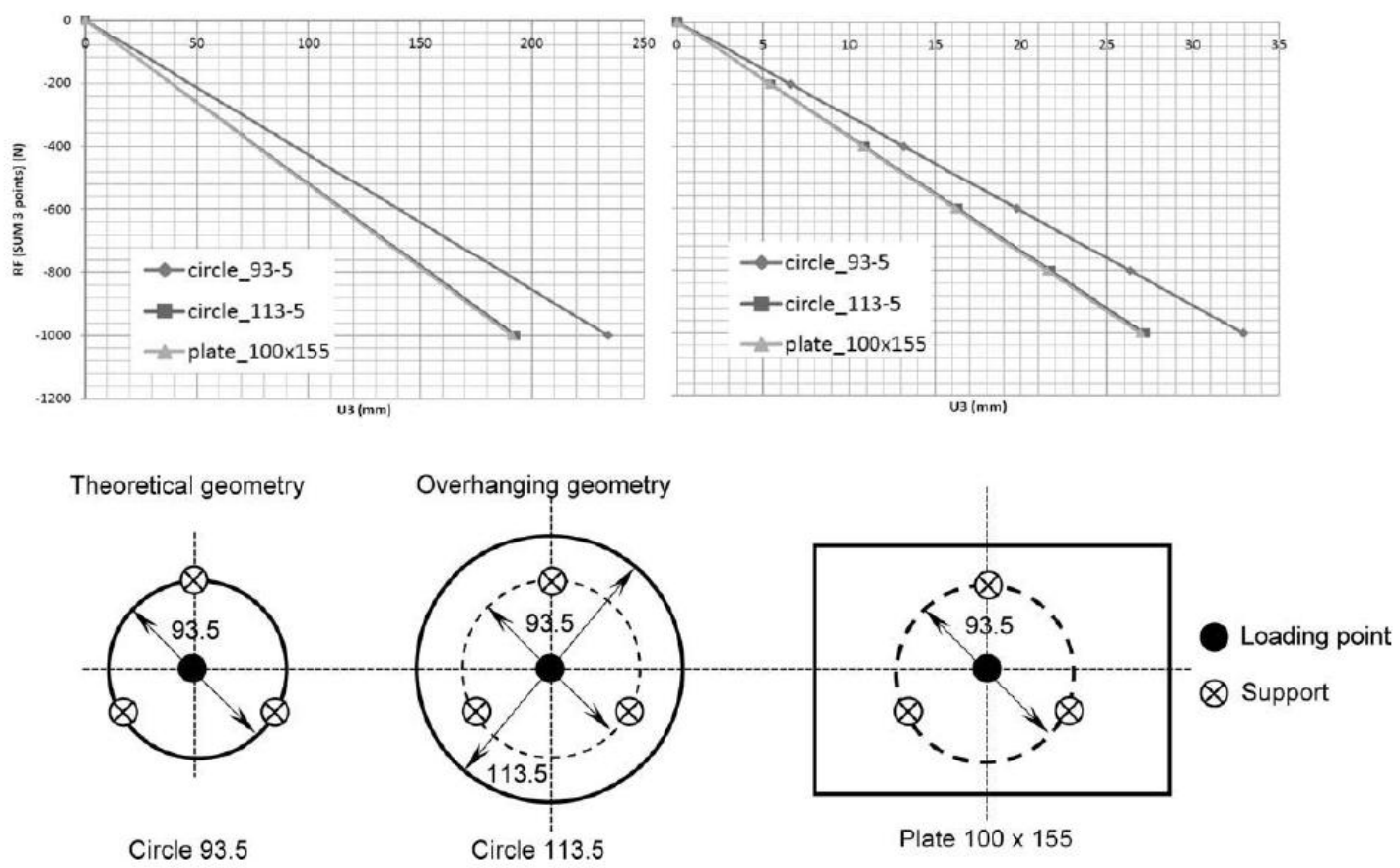

FIG. 4. Simulation results obtained with the ABAQUS software with two circular and one rectangular test specimens subjected to three-point-support flexural tests. 


\section{RESULTS AND DISCUSSION}

\section{SEM Morphology}

Figure 5 shows the morphology revealed by SEM of UDP, CPC, MRB, and NOM composites with two representative HDPE/PA6/YP compositions: without $(80 / 20 / 0)$ and with $10 \%$ compatibilizer (70/20/10). In the UDP samples, long fibrils are observed being nearly parallel to the fracture plane (Fig. 5, panels $1 \mathrm{a}$ and $1 \mathrm{~b}$ ). In the CPCS (Fig. 5, panels $2 a$ and $2 b$ ), the fibrils are aligned as expected in two perpendicular directions, the fibrils with perpendicular orientation to the fracture plane showing a circular cross-section. Figure 5, panels $3 a$ and $3 b$ correspond to the composites produced from MRB. Here, a great variety of fibril cross-section shapes can be observed: rectangular, spherical, or oval, depending on the angle between the fibril and the fracture plane. In the NOM samples, however, oval-shaped entities instead of fibrils are only observed due to the low orientation of the PA6 component.

Generally, in UDP, CPC, and MRB materials compatibilization with YP leads to thinner fibrils. At the same time, YP seems to improve the adhesion between the PA6 and the HDPE components. In the absence of YP (Fig. 1a), the fibrils are completely detached from the matrix whereas in the specimen with compatibilizer (Fig. 1b), they are well embedded, evidencing a better adhesion.

\section{Tensile Properties}

The anisotropic UDP lamina represents the basic building block in composites reinforced by long fibers. Knowing its tensile properties allows their modeling for any kind of laminate composites, produced from two and more such laminae [27]. Therefore, the tensile properties of UDP MFC were characterized as a function of the HDPE/PA6/YP composition, parallel and normal to the direction of the reinforcing microfibrils. Figure 6 shows some typical stress-strain curves of UDP MFC in the longitudinal direction. The 90/10/0 composition containing 10 wt\% PA6 displays a ductile behavior similar to the HDPE matrix. In the two corresponding curves, there exist clear yielding and necking, even though the strain at break $\varepsilon_{\mathrm{br}}$ of the composite (about 100\%) is much smaller than the HDPE alone (about 800\%). The other stress-strain curves show the typical brittle shape of composite materials, with $\varepsilon_{\text {br }}$ not exceeding $30 \%-40 \%$, and $\sigma_{1 \max }$ considerably higher than the matrix. Similar curves were obtained when stretching in a direction transversal to the fiber axis.

The reinforcing effect in the UDP MFC was assessed on the basis of the $E$ and $\sigma_{\max }$ data from the stress-strain curves in comparison with the data of the pure HDPE matrix, or the model predictions based on Eqs. 1-4.
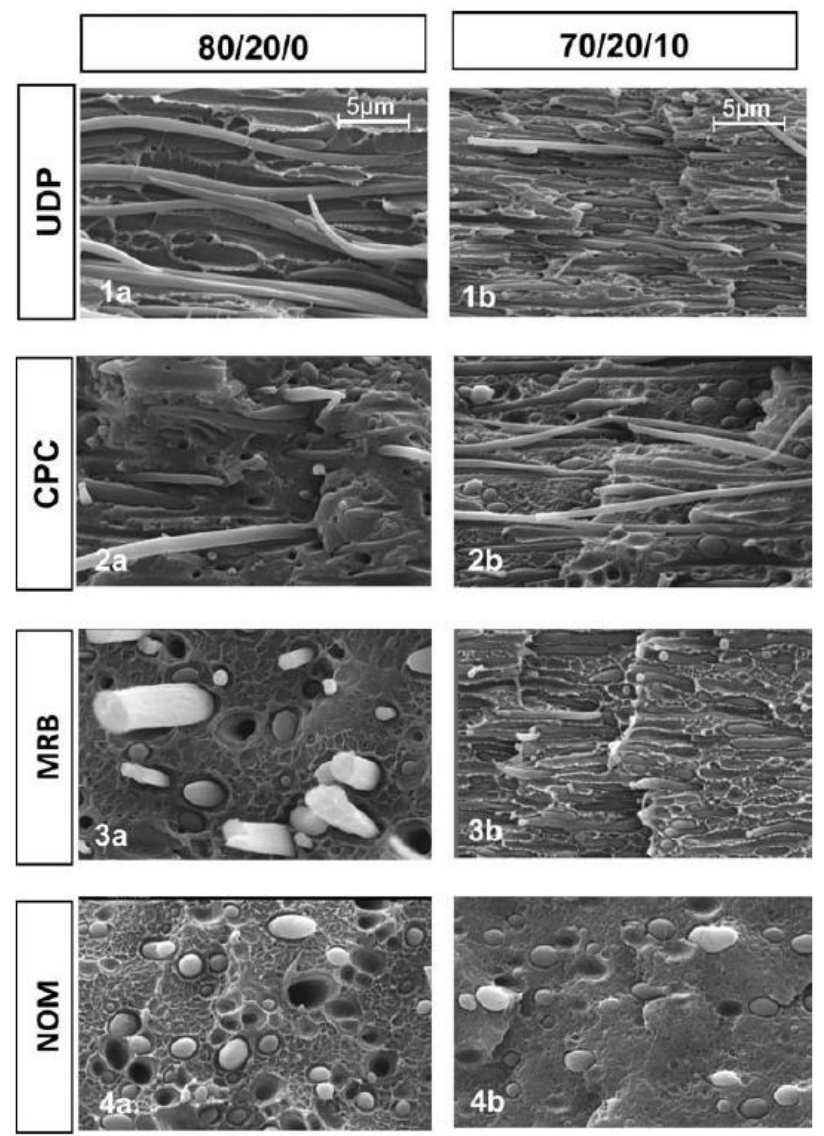

FIG. 5. SEM images of various composites made from two HDPE/ PA6/YP oriented blends [1-4 (a): 80/20/0 wt $\%$; 1-4 (b): 70/20/10 wt $\%$ ]. UDP, unidirectional ply fractured parallel to the fibril direction; CPC, cross-ply composite; MRB, composite from middle-length PA6 bristles with random distribution; NOM, obtained from non-oriented granules of the two blends.

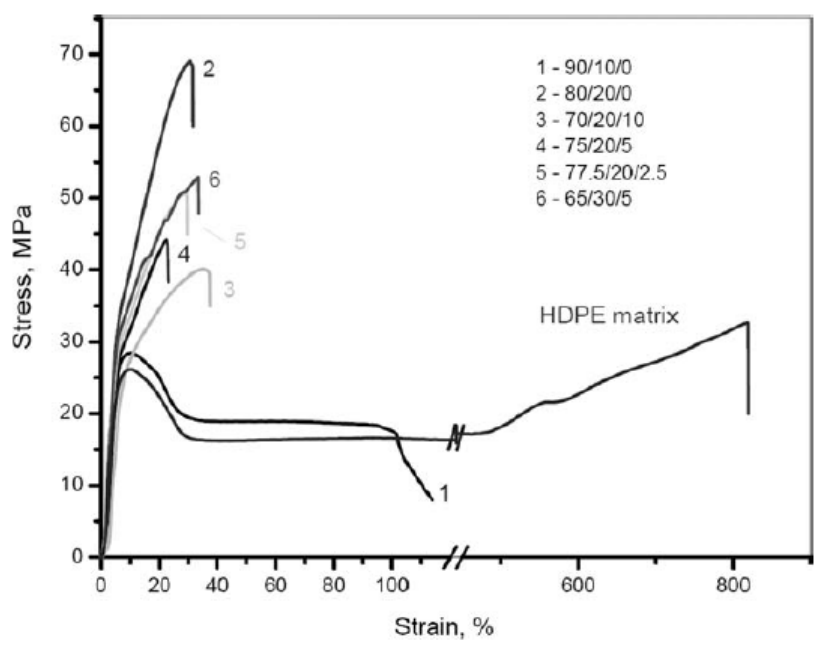

FIG. 6. Representative stress-strain curves of HDPE/PA6/YP UDP MFCs as a function of the sample composition obtained by straining in longitudinal direction. 
Table 2 shows the absolute values of the longitudinal Young modulus $E_{1}$ and the ultimate strength r1max of HDPE/PA6/YP UDP MFC and their relative increases with respect to the HDPE matrix. All MFC compositions show an improvement of $E_{1}$ in the $11 \%-33 \%$ range, the biggest being for composites without compatibilizer and the smallest for the composition with $10 \%$ of Yparex. The $\sigma_{1 \max }$ for all composites grow significantly reaching approximately $60 \mathrm{MPa}$ for the $80 / 20 / 0 \mathrm{MFC}$ or a $120 \%$ of improvement in respect to HDPE. Again, the MFC with the biggest concentration of compatibilizer showed the smallest enhancement of the tensile strength. Similar influence of the compatibilizer has been observed in isotropic HDPE/PA6 blends in the presence of MAH-g-PP copolymer [32]. The authors relate this effect to the low molecular weight of the compatibilizer located at the interface between the two components and acting as a mechanically weak boundary phase.

TABLE 2. Absolute values of the longitudinal Young modulus $E_{1}$ and the ultimate strength $\sigma_{1 \max }$ of HDPE/PA6/YP UDP MFCs and their relative increases in respect to the HDPE matrix.

\begin{tabular}{lcccc}
$\begin{array}{l}\text { Composition } \\
\text { HDPE/PA6/YP, } \\
\text { wt } \%\end{array}$ & $E_{1}, \mathrm{MPa}$ & $\sigma_{1 \max }, \mathrm{MPa}$ & $\Delta E_{1}, \%$ & $\Delta \sigma_{1 \max }, \%$ \\
\hline $100 / 0 / 0$ & $827 \pm 47$ & $26 \pm 1$ & 0 & 0 \\
$90 / 10 / 0$ & $940 \pm 21$ & $27 \pm 1$ & 13.7 & 3.8 \\
$80 / 20 / 0$ & $1095 \pm 52$ & $57 \pm 4$ & 32.4 & 119.2 \\
$70 / 20 / 10$ & $920 \pm 7$ & $37 \pm 2$ & 11.2 & 42.3 \\
$75 / 20 / 5$ & $961 \pm 19$ & $45 \pm 3$ & 16.2 & 73.1 \\
$77.5 / 20 / 2.5$ & $1030 \pm 19$ & $45 \pm 3$ & 24.5 & 73.1 \\
$65 / 30 / 5$ & $1098 \pm 48$ & $52 \pm 8$ & 32.8 & 100.0 \\
$0 / 100 / 0$ oriented & $1830 \pm 31$ & $230 \pm 7$ & - & - \\
\hline
\end{tabular}

Notes: $\quad \Delta E_{1}=\left(E_{1}-E_{\mathrm{HDPE} 1}\right) * 100 / E_{1 \mathrm{HDPE}}, \%, \quad \Delta \sigma_{1}=\left(\sigma_{1}-\right.$ $\left.\sigma_{\mathrm{HDPE}}\right) * 100 / \sigma_{1 \mathrm{HDPE}}, \%$.

Table 3 summarizes the experimental Young moduli and tensile strength data of all HDPE/PA6/YP compositions in the longitudinal and transverse directions and their relative changes with respect to the predictions using Eqs. 1-4. Comparing the predicted and experimental longitudinal $E_{1}$ and $\sigma_{1 \max }$ data, it can be concluded that the rule of the mixtures describes well all HDPE/PA6/YP UDP MFC. The experimental values are close and sometimes higher than predicted, the lower the amount of compatibilizer, the bigger the difference. For example, the $80 / 20 / 0$ system shows the largest positive deviation with $\Delta \mathrm{E}_{1}$ of approximately $10 \%$, whereas the experimental $\mathrm{E}_{1}$ of the $70 / 20 / 10$ composition is smaller than the calculated one with a negative deviation of approximately $28 \%$. The $\Delta \sigma_{1 \max }$ data show the same trend, the non-compatibilized MFCs displaying the biggest positive deviation in the range of $42 \%-46 \%$.

As seen from Table 3, the tensile properties of the HDPE/PA6/YP UDP MFC in the transverse direction do not follow the rule of the mixtures. Most of the $E_{2}$ values are lower than the predictions and are close to HDPE. The $90 / 10 / 0$ composition is the only that shows a positive deviation from the theoretical value. As regards $\sigma_{2 \max }$ it is significantly higher than the expected value of 0.33 r $2 \mathrm{max}$ of HDPE. The positive deviations vary from $46 \%$ for the $80 / 20 / 0 \%$ to $180 \%$ for $90 / 10 / 0$ composition. An exception to this trend is the $65 / 30 / 5$ MFC with very low experimental $E_{2}$ and $\sigma_{2 \max }$ data. It is to be noted that the 90/10/0 laminae display satisfactory tensile properties also in the transversal direction showing a Young modulus approximately $11 \%$ higher than $\mathrm{E}_{*}^{*}$ and experimental tensile strength $180 \%$ higher than $\sigma^{*}{ }_{2 \max }$. The fact that in transversal direction, there are also deviations from the values expected by the theory of the long fiber-reinforced composites, suggests that the respective explanations should be related to the unique structure and morphology of the MFC.

The data in Tables 2 and 3 allow the conclusion that to improve the longitudinal tensile properties of UDP MFC, no compatibilizer or very small amounts of it should be implemented. Compatibilization, however, is needed for better mechanical performance in the UDP MFC in transverse direction and also to enhance the cold drawing processing stage. In addition, both $E_{1}$ and $\sigma_{1 \max }$ of most UDP MFC are higher than predicted using the rule of mixtures. A possible explanation is that the reinforcing PA6 fibrils in the UDP MFC are stiffer and stronger than in the oriented PA6 samples used to calculate the theoretical E and $\sigma$. The tensile properties of the PA6 fibrils in MFC cannot be measured directly because they do not exist as a separate material but instead are formed in situ during the composite preparation. Direct measurements of the aspect ratio of these fibrils are impossible either. However, based on the SEM studies on the morphology development in HDPE/PA6/YP precursors and composites, it can be inferred that in non-compatibilized UDP MFC, the aspect ratio of the reinforcing fibrils is at least 10 times bigger than in those with $10 \%$ of YP [13]. This finding is in good agreement with the superior tensile properties of non compatibilized UDP MFC along the fiber axis. Moreover, the aspect ratio could be even higher than that calculated using data from SEM. We have proved by X-ray analyses that HDPE transcrystallization occurs during the isotropization stage of the MFC preparation. As a result, the reinforcing fibrils consist of a core of PA6 and a shell of oriented, transcrystalline HDPE material. The thickness and the structure of this transcrystalline layer (TCL) depend on both HDPE/PA6 composition and compatibilizer concentration and are closely related to the tensile behavior of the MFC [13].

Table 4 summarizes tensile test data for MRB and NOM composites. The Young moduli and strengths in longitudinal and transverse directions of the MRB MFC are close to one another. Most of the compositions show improvement in the tensile performance when compared with HDPE. The E and $\sigma$ values, however, remain clearly below to those of the UDP lamina in the longitudinal direction. This confirms that the alignment of the fibrils is of major importance for the tensile properties in MFC. 
TABLE 3. Longitudinal and transversal tensile properties of UDP MFCs with various compositions and their relative changes in respect to the predicted values.

\begin{tabular}{|c|c|c|c|c|c|c|c|c|c|c|c|c|}
\hline $\begin{array}{l}\text { Composition, } \\
\text { HDPE/PA YP, } \\
\text { wt } \%\end{array}$ & $\begin{array}{l}\text { Vol. fract. } \\
\text { of PA6, } V_{\mathrm{f}}\end{array}$ & $E_{1}, \mathrm{MPa}$ & $\begin{array}{c}E_{1}^{*} \\
\mathrm{MPa}\end{array}$ & $\Delta E_{1}, \%$ & $\sigma_{1 \max }, \mathrm{MPa}$ & $\begin{array}{c}\sigma_{1 \max }^{*} \\
\mathrm{MPa}\end{array}$ & $\Delta \sigma_{1 \max }, \%$ & $E_{2}, \mathrm{MPa}$ & $E_{2}^{*}, \mathrm{MPa}$ & $\Delta E_{2}, \%$ & $\sigma_{2 \max }, \mathrm{MPa}$ & $\begin{array}{c}\Delta \sigma_{2 \max }, \\
\%\end{array}$ \\
\hline $100 / 0 / 0$ & - & $827 \pm 47$ & - & 0 & $26 \pm 1$ & - & 0 & $851 \pm 32$ & - & 0 & $27.0 \pm 0.4$ & 0 \\
\hline $90 / 10 / 0$ & 0.084 & $940 \pm 21$ & 911 & 3.2 & $27 \pm 1$ & 19 & 42.1 & $962 \pm 65$ & 867 & 11.0 & $25.1 \pm 1.0$ & 181.7 \\
\hline $80 / 20 / 0$ & 0.171 & $1095 \pm 52$ & 999 & 9.6 & $57 \pm 4$ & 39 & 46.2 & $630 \pm 77$ & 913 & -31.0 & $12.6 \pm 0.5$ & 41.4 \\
\hline $70 / 20 / 10$ & 0.171 & $920 \pm 7$ & 998 & -7.8 & $37 \pm 2$ & 39 & -5.1 & $840 \pm 27$ & 913 & -8.0 & $21.4 \pm 0.3$ & 140.2 \\
\hline $77.5 / 20 / 2.5$ & 0.171 & $1030 \pm 19$ & 998 & 3.2 & $45 \pm 3$ & 39 & 15.4 & $855 \pm 24$ & 913 & -6.4 & $21.2 \pm 1.2$ & 137.9 \\
\hline $65 / 30 / 5$ & 0.261 & $1098 \pm 48$ & 1089 & 0.8 & $52 \pm 8$ & 60 & -13.3 & $323 \pm 20$ & 965 & -66.5 & $3.7 \pm 0.4$ & -58.5 \\
\hline $\begin{array}{l}0 / 100 / 0 \\
\quad \text { (oriented) }\end{array}$ & - & $1830 \pm 31$ & - & - & $230 \pm 7$ & - & - & & - & - & - & - \\
\hline
\end{tabular}

$E_{k}^{*}$ and $\sigma_{k \max }^{*}(k=1,2)$ are the theoretical values derived from Eqs. 1 to 4 .

$\Delta E_{k}=\left(E_{k}-E_{k}^{*}\right) \cdot 100 / E_{k}^{*}, \%$ and $\Delta \sigma_{k}=\left(\sigma_{k \max }-\sigma_{k \max }^{*}\right) \cdot 100 / \sigma_{k \max }^{*}, \%$

$\sigma_{2 \max }^{*}=0.33^{*} \sigma_{p \max }=8.91 \mathrm{MPa}$, wherein $\sigma_{p \max }$ is the tensile strength of HDPE in transverse direction.

TABLE 4. Longitudinal and transversal tensile properties of MRB and NOM composites.

\begin{tabular}{|c|c|c|c|c|c|}
\hline HDPE/PA6/YP, wt $\%$ & Vol. fract. of PA6, $V_{\mathrm{f}}$ & $E_{1}, \mathrm{MPa}$ & $E_{2}, \mathrm{MPa}$ & $\sigma_{1 \max }, \mathrm{MPa}$ & $\sigma_{2 \max }, \mathrm{MPa}$ \\
\hline \multicolumn{6}{|c|}{ HDPE/PA6/YP MRB MFC } \\
\hline $100 / 0 / 0$ & - & $827 \pm 47$ & $851 \pm 32$ & $26 \pm 1$ & $27 \pm 1$ \\
\hline $90 / 10 / 0$ & 0.084 & $899 \pm 43$ & $926 \pm 46$ & $24 . \pm .1$ & $23 \pm 1$ \\
\hline $80 / 20 / 0$ & 0.171 & $903 \pm 54$ & $904 \pm 47$ & $24 \pm 6$ & $22 \pm 3$ \\
\hline $70 / 20 / 10$ & 0.171 & $854 \pm 25$ & $891 \pm 7$ & $25 \pm 1$ & $25 \pm 1$ \\
\hline $75 / 20 / 5$ & 0.171 & $898 \pm 70$ & $889 \pm 65$ & $29 \pm 4$ & $25 \pm 1$ \\
\hline $77.5 / 20 / 2.5$ & 0.171 & $886 \pm 34$ & $960 \pm 36$ & $31 \pm 5$ & $24 \pm 3$ \\
\hline $65 / 30 / 5$ & 0.261 & $744 \pm 50$ & $722 \pm 31$ & $16 \pm 2$ & $12 \pm 1$ \\
\hline 0/100/0 oriented & - & $1830 \pm 31$ & - & $230 \pm 7$ & - \\
\hline \multicolumn{6}{|l|}{ HDPE/PA6/YP NOM } \\
\hline $100 / 0 / 0$ & - & $827 \pm 47$ & $851 \pm 32$ & $26 \pm 1$ & $27 \pm 1$ \\
\hline $90 / 10 / 0$ & 0.084 & $906 \pm 53$ & $906 \pm 35$ & $24 \pm 1$ & $25 \pm 1$ \\
\hline $80 / 20 / 0$ & 0.171 & $871 \pm 47$ & $869 \pm 52$ & $22 \pm 1$ & $21 \pm 3$ \\
\hline $70 / 20 / 10$ & 0.171 & $973 \pm 45$ & $1028 \pm 4$ & $29 \pm 1$ & $31 \pm 1$ \\
\hline $75 / 20 / 5$ & 0.171 & $923 \pm 10$ & $986 \pm 33$ & $22 \pm 2$ & $21 \pm 2$ \\
\hline $77.5 / 20 / 2.5$ & 0.171 & $884 \pm 75$ & $893 \pm 46$ & $22 \pm 1$ & $22 \pm 2$ \\
\hline $65 / 30 / 5$ & 0.261 & $772 \pm 59$ & $712 \pm 43$ & $11 \pm 1$ & $9 \pm 1$ \\
\hline $0 / 100 / 0$ isotropic & - & $1110 \pm 40$ & $1080 \pm 35$ & $51 \pm 2$ & $50 \pm 1$ \\
\hline
\end{tabular}

Considering the tensile properties of the NOM composites (Table 4), one should bear in mind that the HDPE matrix is reinforced by microspheres of PA6, similarly to the glass sphere reinforced composites. NOM composites are not MFC, because the reinforcing phase is not fibrilar. The 70/20/10 NOM displays considerable improvement of both Young modulus and tensile strength. This is contrary to what was observed in the respective UDP where the PA6 phase was fibrilar with parallel alignment. At this point, a supposition can be made that when the PA6 is isotropic a better compatibilization can be achieved improving the adhesion at the HDPE/PA6 interface. Another remark is that the 90/10/0 NOM system also displays enhanced modulus values. This was not the case of the conventional melt blended HDPE/PA6 system [31], where a minimum of $20 \%$ of PA6 was necessary to reach some improvement of the tensile properties. The difference can be attributed to the specific processing conditions of the NOM composites, namely to the fact that the matrix isotropization was done at $160^{\circ} \mathrm{C}$, i.e., far below the PA6 melting point. In such a way, PA6 undergoes annealing that leads to higher crystallinity and increase of the $\alpha$-PA6 polymorph content, observed with isotropic and oriented PA6 samples annealed at $1608 \mathrm{C}[25,26]$.

Figure 7 shows a direct comparison of the longitudinal tensile properties of all the UDP, MRB, and NOM composites as a function of their HDPE/PA6/YP composition. It can be seen (Fig. 7a) that most all of the composites display $E_{1}$ higher than HDPE, the only exception being the 65/30/5 system, where only the UDP lamina shows improved tensile stiffness. In the case of the $90 / 10 / 0$ composites, there is no statistically significant difference between the moduli of UDP, MRB, and NOM, i.e., the type of PA6 reinforcement (fibrilar or isotropic) and the alignment of the fibrils do not influence the stiffness. Considering the compositions with $20 \%$ PA6, one can assess the influence of these two parameters, as well as that of the compatibilizer. Clear enhancement of the modulus is registered only where the reinforcements are aligned fibrils-in the UDP laminae. Within the 20\% PA6 series, high moduli are observed either without or at low YP concentrations. When the reinforcing component is isotropic (NOM), the trend is inversed. In this case, the higher compatibilizer concentration favors the stiffness. 

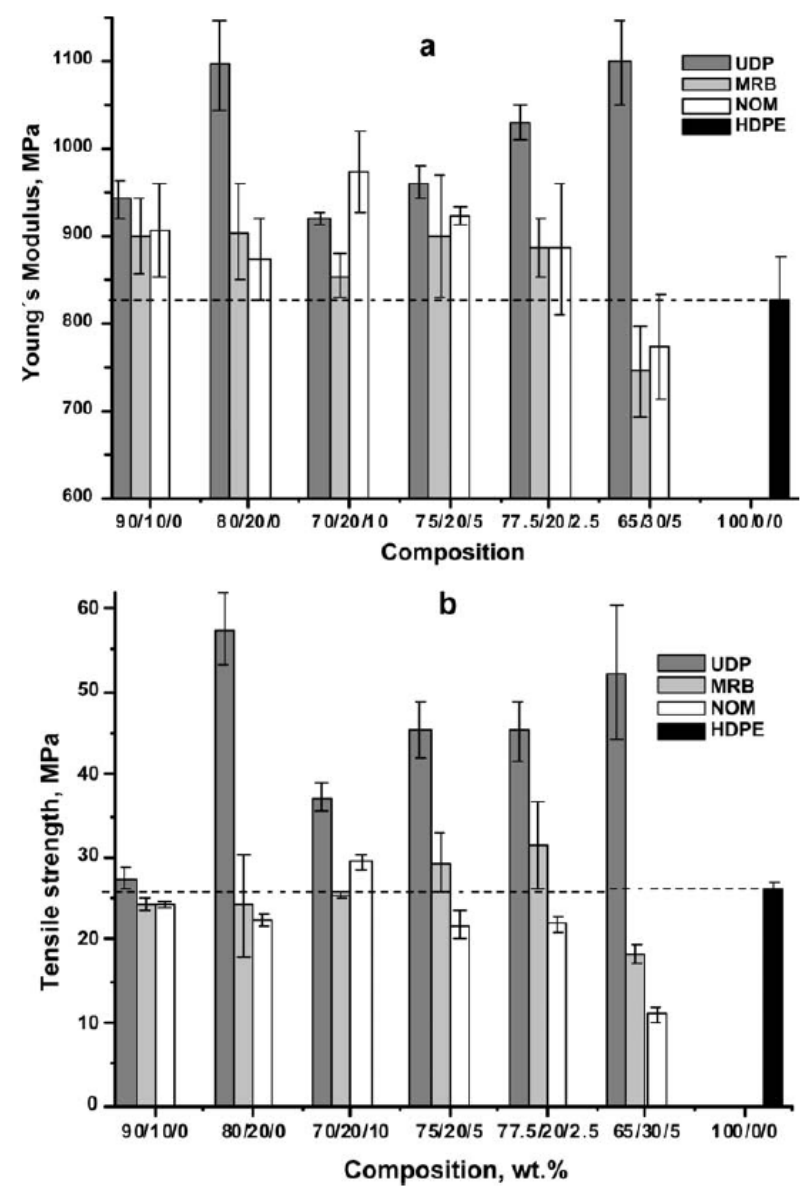

FIG. 7. Longitudinal tensile properties of HDPE/PA6/YP UDP, MRB, and NOM composites and the HDPE matrix: (a) $E_{l}$ and (b) $\sigma_{1 \max }$.

Apparently, the compatibilizing effect is better expressed when the PA6 reinforcement is isotropic. This is in good agreement with morphological studies of various
HDPE/PA6/YP MFC after selective dissolution of the HDPE matrix [13]. The SEM observation showed that when the reinforcement is isotropic (globular), in the presence of YP, the matrix selective dissolution is hampered, which shows better chemical interaction at the HDPE/PA6 interface. As far as the tensile strength values are concerned (Fig. 7b), a clear increase of $\sigma_{1 \max }$ is observed only in the UDP MFC. The compatibilizer concentration influences the strength in the same way as the stiffness. The systems without compatibilizer show an improvement of $\sigma_{1 \max }$ of above $100 \%$. In the isotropic MRB and NOM composites, the strength data are close or even worse than those of the matrix. Therefore, to obtain in situ MFC of higher strength and stiffness one should consider the preparation of laminates with several UDP.

\section{Flexural Tests in CPC Laminates}

In practice, in very few cases, materials work in tensile mode, more often, they are subjected to flexure or impact. At the same time, fiber-reinforced composites are usually applied as laminates with different orientation and alignment of the fibrous reinforcement. That is why crossply laminates were produced and used to study their flexural stiffness and impact resistance.

The flexural data for HDPE/PA6/YP CPC MFC are summarized in Table 5 . All composites show a notable improvement of the flexural performance with $C_{R}$ values of 2.3-2.6 GPa, i.e., well above the HDPE matrix value of 1.5 $\mathrm{GPa}$. Thus, for the CPC laminates, the IF varies between $55 \%$ for $70 / 20 / 10$ system to $78 \%$ for $80 / 20 / 0$, whereby increasing the concentration of YP resulted in smaller $C_{R}$. The 80/20/0 CPC MFC shows the higher increase of $C_{R}$. The same system as a UDP lamina had the best performance in tension too, with improvements in the Young modulus and

TABLE 5. Flexural properties of CPC, MRB, and NOM composites.

\begin{tabular}{|c|c|c|c|c|}
\hline Composition, HDPE/PA6/YP, wt $\%$ & Slope, $S_{\mathrm{p}}, \mathrm{N} / \mathrm{mm}$ & Average thickness, $h, \mathrm{~mm}$ & Flexural stiffness, $C_{R}$, GPa & Improvement factor, IF, $\%$ \\
\hline $100 / 0 / 0$ & 11.361 & 2.002 & $1.478 \pm 0.057$ & 0 \\
\hline \multicolumn{5}{|l|}{ HDPE/PA6/YP CPC } \\
\hline $90 / 10 / 0$ & 11.844 & 1.705 & $2.493 \pm 0.148$ & 68.7 \\
\hline $80 / 20 / 0$ & 17.262 & 1.893 & $2.624 \pm 0.245$ & 77.6 \\
\hline $70 / 20 / 10$ & 11.366 & 1.730 & $2.294 \pm 0.224$ & 55.2 \\
\hline $75 / 20 / 5$ & 12.868 & 1.736 & $2.564 \pm 0.146$ & 73.4 \\
\hline $77.5 / 20 / 2.5$ & 13.543 & 1.758 & $2.595 \pm 0.109$ & 75.6 \\
\hline $65 / 30 / 5$ & 14.621 & 1.823 & $2.516 \pm 0.191$ & 70.3 \\
\hline \multicolumn{5}{|l|}{ HDPE/PA6/YP MRB } \\
\hline $90 / 10 / 0$ & 12.946 & 1.774 & $2.425 \pm 0.182$ & 64.1 \\
\hline $80 / 20 / 0$ & 15.524 & 1.916 & $2.302 \pm 0.148$ & 55.8 \\
\hline $70 / 20 / 10$ & 13.198 & 1.766 & $2.503 \pm 0.051$ & 69.3 \\
\hline $75 / 20 / 5$ & 13.370 & 1.845 & $2.216 \pm 0.175$ & 50.0 \\
\hline $77.5 / 20 / 2.5$ & 12.559 & 1.791 & $2.282 \pm 0.085$ & 54.4 \\
\hline $65 / 30 / 5$ & 16.800 & 1.862 & $2.723 \pm 0.376$ & 84.2 \\
\hline \multicolumn{5}{|l|}{ HDPE/PA6/YP NOM } \\
\hline $90 / 10 / 0$ & 12.365 & 1.736 & $2.464 \pm 0.110$ & 66.8 \\
\hline $80 / 20 / 0$ & 11.448 & 1.740 & $2.267 \pm 0.176$ & 53.4 \\
\hline $70 / 20 / 10$ & 10.521 & 1.710 & $2.198 \pm 0.098$ & 48.7 \\
\hline $75 / 20 / 5$ & 11.042 & 1.719 & $2.271 \pm 0.024$ & 53.6 \\
\hline $77.5 / 20 / 2.5$ & 11.192 & 1.719 & $2.301 \pm 0.072$ & 55.7 \\
\hline $65 / 30 / 5$ & 18.919 & 2.017 & $2.408 \pm 0.138$ & 62.9 \\
\hline
\end{tabular}


tensile strength of 32 and 119\%, respectively. In the HDPE/PA6/YP MRB series, it is the 65/30/5 composition that shows the highest $C R$. This seems to be related to the largest amount (30\%) of randomly arranged short PA6 reinforcing fibrils. Within the MRB samples containing $20 \%$ PA6, the composition with $10 \%$ compatibilizer displays unusually good flexural characteristics. Similarly to the CPC and MRB, all NOM compositions also showed better performance than the HDPE matrix.

As seen from the comparison in Fig. 8, there is no statistically significant difference in the flexural stiffness data of CPC, MRB, and NOM composites, especially in samples containing $10 \%$ of PA6. This means that for the flexural properties, it does not really matter if the reinforcement is isotropic or oriented. The alignment of the PA6 fibrils does not seem to be important in this case either. It should be pointed out that only $10 \%$ of PA6 is sufficient to impart a notable flexural stiffness to the HDPE matrix, the improvement being in the range of $60 \%-70 \%$. Higher improvement factors of $70 \%-80 \%$ were observed in the CPC laminates containing 20\% PA6, in the absence or at low concentration of the YP compatibilizer. The increase of the YP concentration up to $10 \%$ causes deterioration of the flexural behavior of the CPC composites. The composition with 30\% PA6 also leads to an improvement in the flexural properties comparable to that with $20 \%$ reinforcement.

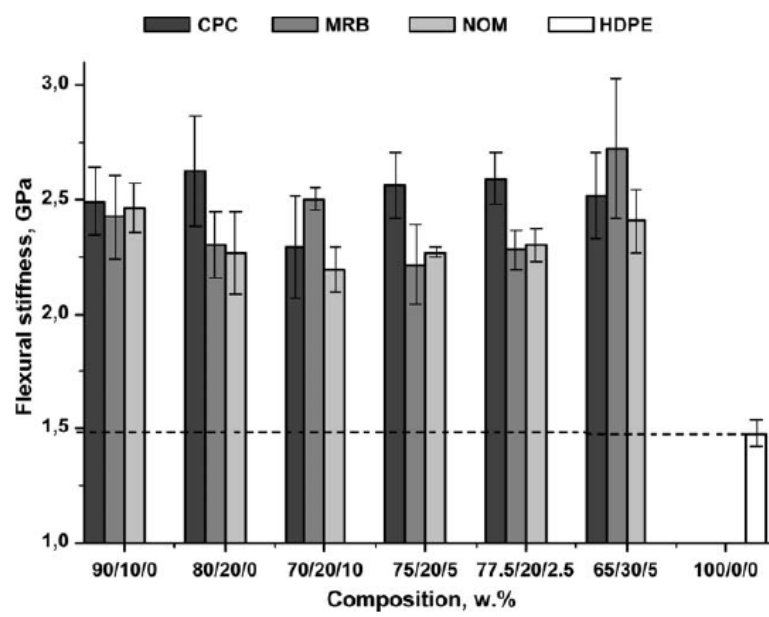

FIG. 8. Comparative chart of the flexural stiffness of all HDPE/PA6/ YP composites studied.

\section{Impact Resistance Tests of HDPE/PA6/YP Laminates}

The peak and total energies per thickness for all sample types are represented in Fig. 9 as a function of the composition. In the case of CPC and MRB MFC where the reinforcement is fibrilar, (Fig. 9a and b), the peak impact energy is lower than for HDPE. The total impact energy, however, is much higher than the matrix. This means that in CPC and MRB, the failure starts at lower energy levels, but the crack propagation before the total failure requires more energy. It can be noted that the $80 / 20 / 0$ and 77.5/20/2.5 CPC composites require a considerable
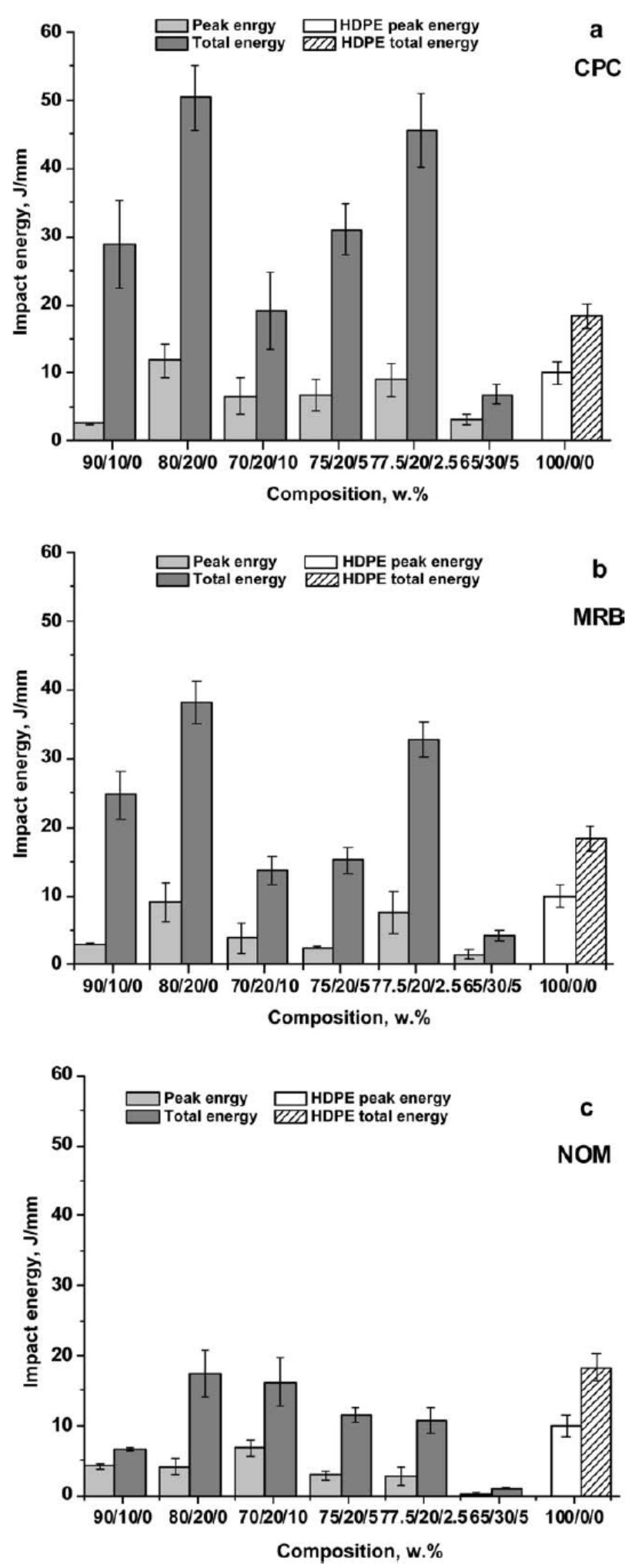

FIG. 9. Impact energy/mm of HDPE/PA6/YP composites with various compositions and reinforcement: (a) cross-ply laminate MFC (CPC); (b) MFC with middle length randomly distributed bristles (MRB); (c) composite prepared by NOM.

increase of the total energy, whereas their peak energies are only slightly above the matrix. It seems that in CPC and MRB MFC $20 \%$ of PA6 is the optimal concentration; increasing the PA6 phase to $30 \%$ has a negative effect and 
keeping it as low as $10 \%$ is not enough, as far as the peak energy is concerned. It is noteworthy that the total impact strength is quite sensitive to the YP content, decreasing as YP increases. These findings agree with the tensile studies, where the MFC containing 20\% of PA6 without or with minimum amount of YP performed the best. The NOM composites where the reinforcing PA6 entities are globular show impact characteristics significantly lower than the HDPE matrix (Fig. 9c). Hence, it is the fibrillar morphology of the PA6 phase in CPC and MRB composites that favors the impact properties.

We believe that the formation of oriented HDPE TCL on the PA6 fibrils proved in the case of HDPE/PA6/YP UDP [13] will also take place in the CPC and MRB laminates thus affecting their mechanical performance. Our method of detection and assessment of the transcrystalline HDPE by $\mathrm{X}$-ray techniques is based on the uniaxial orientation of the fibrils. Any other orientation of the latter (as in CPC or $\mathrm{MRB}$ ) does not produce unambiguous results allowing for a direct structure-property relation.

\section{CONCLUSIONS}

- Depending on the PA6 and YP amounts, the HDPE/ PA6/YP MFC studied in the form of UDP, $C P C$, and MRB showed better mechanical performance than the HDPE matrix in terms of their tensile, flexural and impact properties. Twenty percent of PA6 reinforcement seems to be the optimal concentration.

- The fibrilar morphology of the PA6 reinforcement is needed for major improvement of all mechanical properties.

- In composites with fibril reinforcement (UDP, CPC, and $\mathrm{MRB}$ ), the Yparex compatibilizer has a negative effect on the mechanical properties in tensile, flexural, and impact modes. In NOM where the reinforcement is globular, the effect is reversed.

These conclusions need structural explanations that were looked for in our recent studies on the MFC structure-mechanical properties relationship. As already mentioned above, in the UDP laminae, the better mechanical properties of the non-compatibilized HDPE/PA6 MFC should be related to the significantly larger aspect ratio of the reinforcing PA6 fibrils (_70) when compared with the case of compatibilized MFC (_7.0) [13] The same work also presents proofs that a transcrystalline oriented polyethylene layer (TCL) is formed as a coating on each PA6 fibril. Furthermore, our simultaneous synchrotron X-ray/straining experiments of HDPE/PA6 oriented blend precursors without compatibilizer displayed affine deformation of the macro- and nanostructure resulting in superior tensile properties than in the respective compatibilized samples with chemical bonds between the PA6 and HDPE entities [33]. Our recent study on the Yparex compatibilizer [34] showed that it represents a maleinized linear low-density polyethylene (LLDPE) with a number average molecular weight being significantly lower than that of the matrix HDPE and with much higher index of polydispersity. Based on the electron microscopy and synchrotron X-ray scattering data of MFC in $[13,33,34]$, it can be concluded that in compatibilized MFC, it is the LLDPE from the compatibilizer that forms the $\mathrm{TCL}$ and thus builds the mechanically weaker boundary layer.

\section{REFERENCES}

1. Z. Denchev and N. Dencheva, Polym. Int., 57, 11 (2008).

2. M. Evstatiev and S. Fakirov, Polymer, 33, 877 (1992).

3. S. Fakirov, M. Evstatiev, and J.M. Schultz, Polymer, 34, 4669 (1993).

4. S. Fakirov, M. Evstatiev, and S. Petrovich, Macromolecules, 26, 5219 (1993).

5. M. Evstatiev, S. Fakirov, G. Bechtold, and K. Friedrich, Adv. Polym. Technol., 19, 249 (2000).

6. S. Fakirov, H. Kamo, M. Estatiev, and K. Friedrich, J. Macromol. Sci. Phys., 43, 775 (2005).

7. K. Friedrich, M. Evstatiev, S. Fakirov, O. Evstatiev, M. Ishii and M. Harrass, Compos. Sci. Technol., 65, 107 (2005).

8. A. Monticciolo, P. Cassagnau, and A. Michel, Polym. Eng. Sci., 38, 1882 (1998).

9. M. Evstatiev, S. Fakirov, B. Krasteva, K. Friedrich, J.A. Covas, and A.M. Cunha, Polym. Eng. Sci., 42, 826 (2002).

10. Z.M. Li, M.B. Yang, R. Huang, W. Yang, and J.M. Feng, Polym. Plast. Technol. Eng., 41, 19 (2002).

11. Z.M. Li, W. Yang, B.H. Xie, K.Z. Shen, R. Huang, and M.B. Yang, Macromol. Mater. Eng., 289, 349 (2004).

12. Z. Denchev, M.J. Oliveira, and O.S. Carneiro, J. Macromol. Sci. Phys., B43, 143 (2004).

13. N. Dencheva, M.J. Oliveira, O.S. Carneiro, A.S. Pouzada, and Z. Denchev. J. Appl. Polym. Sci., 115, 2918 (2010)

14. S. Filippi, V. Chiomo, J. Polacco, M. Paci, M.I. Minkova and P. Magagnini, Macromol. Chem. Phys., 203, 1512 (2002).

15. W. Loyens and G. Groeninckx, Macromol. Chem. Phys., 203, 1702 (2002).

16. S. Fakirov, H. Kamo, M. Estatiev, and K. Friedrich, J. Macromol. Sci. Phys., B43, 775 (2004).

17. Z.M. Li, B.L. Li, K.Z. Shen, W. Yang, R. Huang, and M.B. Yang, Macromol. Rapid. Commun., 25, 553 (2004).

18. D. Sapoundjieva, Z. Denchev, M. Evstatiev, S. Fakirov, N. Stribeck, and M. Stamm, J. Mater. Sci., 34, 3063 (1999).

19. S. Fakirov, M. Evstatiev, and K. Friedrich, "Nanostructured Polymer Composites from Polymer Blends: Morphology and Mechanical Properties," in Handbook of Thermoplastic Polyesters, S. Fakirov, Ed., Wiley-VCH, Weinheim (2002).

20. C. Fuchs, D. Bhattacharyya, and S. Fakirov, Compos. Sci. Technol., 66, 3161 (2006).

21. Z.M. Li, B.H. Xie, R. Huang, X.P. Fang, and M.B. Yang, Polym. Eng. Sci., 44, 2165 (2004). 
22. L. Fasce, R. Seltzer, P. Frontini, V.J. RodriguezPita,E.B.A.V. Pacheco, and M.L. Dias, Polym. Eng. Sci., 45, 354 (2005).

23. O. Evstatiev, F. Oster, K. Friedrich, and S. Fakirov, Int. J. Polym. Mater., 53, 1071 (2004).

24. J.E. Mark, Polymer Data Handbook, Oxford University Press, New York, 180 (1999).

25. N. Dencheva, Z. Denchev, M.J. Oliveira, and S.S. Funari. J. Appl. Polym. Sci., 103, 2242 (2007).

26. N. Dencheva, T. Nunes, M.J. Oliveira, and Z. Denchev, Polymer, 46, 887 (2005).

27. P. C. Powell, Engineering With Fiber-Polymer Laminates, Chapman \& Hall, London, UK (1994).

28. J.P. Nunes, A.S. Pouzada, and C.A. Bernardo, Polym. Test., 21, 27 (2002).

29. A.S. Pouzada and M.J. Stevens, Plast. Rubber. Compos. Process. Appl., 4, 181 (1984).

30. ABACUS CAE 6.8 Version. Dassault Syste`mes, Lowell, MA (2004).

31. L.T. Pick and E. Harkin-Jones, Polym. Eng. Sci., 43, 905 (2004).

32. M. Palabiyik and S. Bahadur, Wear, 246, 149 (2000).

33. Z. Denchev, N. Dencheva, S.S. Funari, M. Motovilin, T. Schubert, and N. Stribeck, J. Polym. Sci. Part. B: Polym. Phys., 48, 237 (2010)

34. N. Dencheva, Z. Denchev, M.J. Oliveira, and S.S. Funari, Macromolecules., 43, 4715 (2010) 